\title{
Evidence that $p$-Fluorophenylalanine has a Direct Effect on Tubulin in Aspergillus nidulans
}

\author{
By N. RONALD MORRIS AND C. ELIZABETH OAKLEY \\ Department of Pharmacology, College of Medicine and Dentistry of New Jersey, \\ Rutgers Medical School, P.O. Box 101, Piscataway, New Jersey 08854, U.S.A.
}

(Received 8 February 1979)

\begin{abstract}
Three temperature-sensitive alleles of benA (benA11,17 and 21) confer resistance to growth inhibition by $p$-fluorophenylalanine (FPA). FPA resistance cosegregates with the ben $A$ gene. Two back-mutations in ben $A$ which cause loss of temperature sensitivity cause loss of FPA resistance, and two indirect suppressors of benA temperature sensitivity also cause FPA resistance to be lost. These results indicate that FPA resistance is an intrinsic property of the ben $A$ mutations. The intracellular phenylalanine concentrations of these strains are normal as is their ability to take up phenylalanine from the medium. We conclude that FPA must inhibit growth and cause non-disjunction by a direct effect on the polymerization of tubulin.
\end{abstract}

\section{INTRODUCTION}

Benomyl and $p$-fluorophenylalanine (FPA) are commonly used in fungal genetics to induce loss of chromosomes from diploids during parasexual mapping of genes to chromosomes (Lhoas, 1961; Hastie, 1970). Benomyl is known to interact with the $\beta$-subunit of tubulin at a site closely related to the colchicine binding site and presumably causes chromosome loss by interfering with the polymerization or function of microtubules in the mitotic spindle (Davidse, 1973; Davidse \& Flach, 1977; Sheir-Neiss et al., 1978). The mechanism by which FPA causes chromosome loss has not yet been explained.

During the course of our studies of benomyl-resistant mutants of Aspergillus nidulans (Sheir-Neiss et al., 1978), we noted that three strains with mutations (benA11,17 and 21) in the ben $A$ gene which were temperature-sensitive for growth (Morris et al., 1979) were also cross-resistant to FPA. We have shown elsewhere that ben $A$ is a structural gene for $\beta$ tubulin (Sheir-Neiss et al., 1978) and that the temperature sensitivity of the benA11, benA17 and benA21 alleles is an intrinsic property of these ben $A$ mutations (Morris et al., 1979). In this paper, we demonstrate that resistance to FPA is another intrinsic property of the benA11, 17 and 21 mutations. From this we conclude that FPA probably affects mitosis by a direct effect on tubulin.

\section{METHODS}

Strains. B3 (biAl acrAl), BEN1 to 25 (benA alleles benA3 to 27 biAl acrAl) and BEN28 (benA28 sUAladE20 yA2 adE20 acrA1 sB3 riboB2) were obtained from Dr J. M. Van Tuyl. FGSC 99 (suAladE20 adE20 yA2 pabaAl proAl acrAI), FGSC 73 (pabaA1 yA2 adE20 acrA1 phenA2 pyroA4 lysB5 sB3 riboB2) and FGSC 273 ( adF17 pabaA1 y $A 2$ fpaD11) were obtained from the Fungal Genetics Stock Center at Arcata, California, U.S.A.

Genetic methods and media. These were standard for A. nidulans (Pontecorvo et al., 1953; Clutterbuck, 1974). Back-mutations and strains carrying suppressor mutations were isolated as previously described (Morris et al., 1979). 
Chemicals. Benomyl was a gift from E. I. du Pont de Nemours and Co. FPA was purchased from Calbiochem.

Amino acid analysis. Cultures of $\mathrm{B} 3$, and strains carrying ben $\mathrm{A} 11,17$ and 21 , were grown at $37^{\circ} \mathrm{C}$ for $16 \mathrm{~h}$ from an inoculum of $3 \times 10^{6}$ conidia $\mathrm{ml}^{-1}$, washed with cold saline and harvested by filtration. The mycelia were resuspended in $1 \mathrm{ml}$ of cold $10 \%(\mathrm{w} / \mathrm{v})$ sulphosalicylic acid and homogenized thoroughly with a Teflon-glass homogenizer. The debris was removed by centrifugation. The supernatant liquid was then adjusted to pH 2.2 with $\mathrm{NaOH}$, centrifuged to remove the precipitate and $100 \mu \mathrm{l}$ samples were applied to a Beckmann $121 \mathrm{MB}$ amino acid analyser. Peaks were identified by their time of elution and the phenylalanine peak was verified by addition of excess phenylalanine to one sample. The peaks were cut out and weighed to determine their relative areas.

\section{RESULTS}

\section{Strains carrying benA11, 17 and 21 are cross-resistant to FPA}

Twenty-six ben $A$ mutants of $A$. nidulans originally isolated by Van Tuyl (1977) were tested for their ability to grow on FPA at $130 \mu \mathrm{g} \mathrm{ml}^{-1}$, a concentration which is inhibitory to the wild-type parental strain B3. Many of the mutants were moderately resistant to FPA (Table 1), the most resistant being three temperature-sensitive ben $A$ mutants carrying benA11, 17 and 21 (Fig. 1). Whereas B3 showed 50\% growth inhibition (LD $\left.\mathrm{LD}_{50}\right)$ on $88 \mu \mathrm{g}$ FPA ml ${ }^{-1}$, the benA11, 17 and 21 strains were $50 \%$ inhibited at 310,220 and $225 \mu \mathrm{g}$ FPA $\mathrm{ml}^{-1}$, respectively.

To determine whether the FPA cross-resistance of these benomyl-resistant strains was related to mutation in the ben $A$ gene, the three strains were outcrossed to $A$. nidulans strain

Table 1. FPA and temperature sensitivity of benA strains

\begin{tabular}{|c|c|c|c|}
\hline Strain & Genotype & $\begin{array}{c}\mathrm{LD}_{50} \text { FPA } \\
\left(\mu \mathrm{g} \mathrm{ml}^{-1}\right)\end{array}$ & $\begin{array}{l}\text { Temperature } \\
\text { sensitivity* }\end{array}$ \\
\hline B3 & ben $A^{+}$biAl acr $A 1$ & 88 & wt \\
\hline BEN1 & benA3 biAI acrAl & 135 & $w \mathrm{t}$ \\
\hline BEN2 & benA4 biAl acrAI & 150 & wt \\
\hline BEN3 & benA5 biAl acr $A 1$ & 72 & wt \\
\hline BEN4 & benA6 biA1 acrA1 & 170 & wt \\
\hline BEN5 & benA7 biAl acrAl & 78 & wt \\
\hline BEN6 & ben $A 8$ biAl acr $A 1$ & 175 & wt \\
\hline BEN7 & ben $A 9$ biAl acrA1 & 160 & CS \\
\hline BEN8 & benA10 biAl acrAl & 120 & wt \\
\hline BEN9 & benAll biAl acrAI & 310 & TS \\
\hline BEN10 & benA12 biAl acr $A 1$ & 200 & wt \\
\hline BEN11 & benA13 biAl acrAl & 105 & wt \\
\hline BEN12 & ben $A 14$ biAl acr $A 1$ & 60 & wt \\
\hline BEN13 & benA15 biAl acr $A 1$ & 170 & wt \\
\hline BEN14 & benAl6 biAl acrAl & 170 & wt \\
\hline BEN15 & ben $A 17$ biAl acrAl & 220 & $\mathrm{CS}, \mathrm{TS}$ \\
\hline BEN16 & benA18 biAl acrA1 & 108 & wt \\
\hline BEN17 & ben $A 19$ biAl acr $A 1$ & 125 & wt \\
\hline BEN18 & ben $A 20$ biAl acr $A 1$ & 68 & wt \\
\hline BEN19 & benA21 biAI acr $A 1$ & 225 & CS, TS \\
\hline BEN20 & ben $A 22$ biAl acr $A 1$ & 72 & wt \\
\hline BEN21 & ben $A 23$ biA1 acrAl & 128 & CS \\
\hline BEN22 & ben $A 24$ biAl acrA1 & 145 & wt \\
\hline BEN23 & ben $A 25$ biAl acrA1 & 62 & wt \\
\hline BEN24 & ben $A 26$ biAl acr $A 1$ & 165 & - \\
\hline BEN25 & ben $A 27$ biAl acr $A 1$ & 75 & wt \\
\hline BEN28 & $\begin{array}{c}\text { benA28 suAladE20 } \\
\text { y } A 2 \text { adE20 acr } A 1\end{array}$ & & \\
\hline \multirow{3}{*}{ FGSC 273} & $S B 3$ riboB2 & 30 & wt \\
\hline & fpaD11 adF17 pabaA1 & & \\
\hline & $y A 2$ & $>1000 \dagger$ & - \\
\hline
\end{tabular}

* CS, Poor growth relative to wild-type at $20^{\circ} \mathrm{C}$; TS, poor growth relative to wild-type at $44{ }^{\circ} \mathrm{C}$; wt, normal growth response to high and low temperatures.

$\dagger$ From Sinha (1969). 


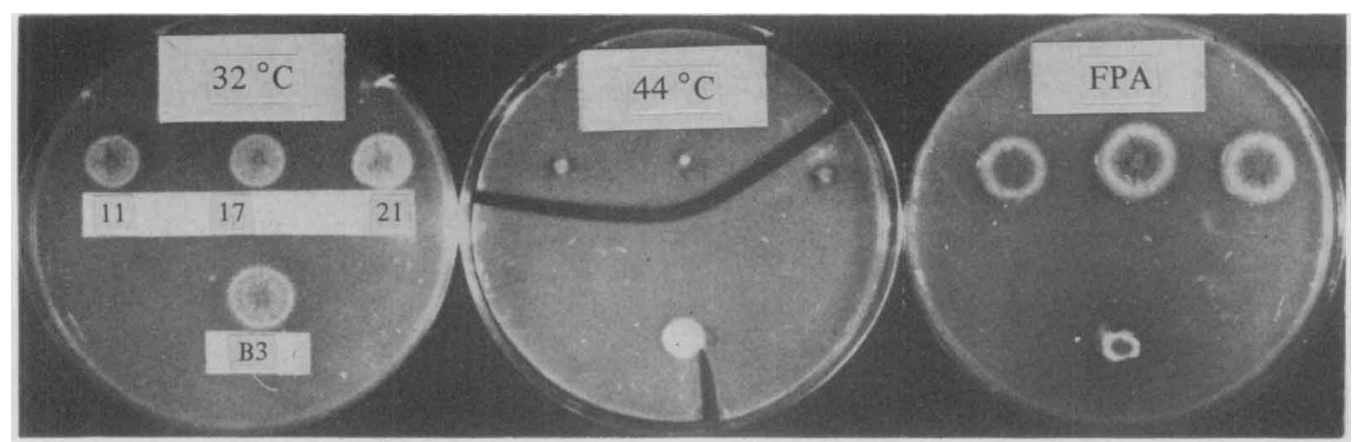

Fig. 1. Effect of high temperature $\left(44^{\circ} \mathrm{C}\right)$ and $p$-fluorophenylalanine (FPA, $130 \mu \mathrm{g} \mathrm{ml}^{-1}$ ) on the growth of benA11, 17 and 21 strains and the parental strain B3 $\left(\right.$ ben $\left.A^{+}\right)$. Plates containing YAG $[0.5 \%(\mathrm{w} / \mathrm{v})$ yeast extract (Difco), $2 \%(\mathrm{w} / \mathrm{v})$ agar, $2 \%(\mathrm{w} / \mathrm{v})$ glucose] were inoculated with conidia and incubated for $3 \mathrm{~d}\left(32^{\circ} \mathrm{C}\right.$ and $44^{\circ} \mathrm{C}$ plates) or $5 \mathrm{~d}$ (FPA plate).

Table 2. Cosegregation of benomyl resistance and FPA resistance among the progeny of FGSC $99 \times$ ben A11, 17 or 21

The results indicate the number of progeny in each class: $\mathrm{R}$, resistant; $\mathrm{S}$, sensitive.

\begin{tabular}{|c|c|c|c|c|c|c|}
\hline & ben $A$ & C 99 & ben $A$ & C 99 & $\operatorname{ben} A$ & C 99 \\
\hline & Ben $R$ & Ben $S$ & Ben $\mathbf{R}$ & Ben $S$ & Ben $R$ & $\operatorname{Ben} S$ \\
\hline $\begin{array}{l}\text { FPAS } \\
\text { FPA R }\end{array}$ & $\begin{array}{r}0 \\
38\end{array}$ & $\begin{array}{r}58 \\
0\end{array}$ & $\begin{array}{r}0 \\
34\end{array}$ & $\begin{array}{r}42 \\
0\end{array}$ & $\begin{array}{r}0 \\
58\end{array}$ & $\begin{array}{r}36 \\
0\end{array}$ \\
\hline
\end{tabular}

FGSC 99 which is sensitive to both FPA and benomyl. Progeny from these crosses were then tested for the presence of the ben $A$ mutation by their ability to grow with an inhibitory concentration of benomyl $\left(2 \cdot 4 \mu \mathrm{g} \mathrm{ml}^{-1}\right)$ and for the presence of the mutation conferring FPA resistance by their ability to grow on FPA. FPA resistance cosegregated perfectly with the ben $A$ gene among the segregants of the outcrosses of benA11, 17 and 21 to FGSC 99 (Table 2). Thus FPA resistance appears to be caused either by the mutations benA11, 17 and 21 or by mutations very closely linked to the ben $A$ gene. Compelling evidence that FPA resistance is an intrinsic property of benA17 and benA21 has been provided by studies of revertants of strains carrying these mutations.

\section{Revertants of benA11, 17 and 21 strains}

The benA11, 17 and 21 strains are not only benomyl-resistant and FPA-resistant but they are also temperature-sensitive (ts ${ }^{-}$) for growth. The benA17 and 21 strains are also coldsensitive for growth. Because it is not possible to select revertants from drug resistance to drug sensitivity, we used the temperature sensitivity of these strains to select revertants from ts $^{-}$to ts $^{+}$. The methods used for selecting these revertants have been described elsewhere (Morris et al., 1979). Three ts ${ }^{+}$revertants were isolated from benA11, nine from benA17 and six from benA21. Each of the ts ${ }^{+}$revertants was then classified as being due to a backmutation in ben $A$ or to a suppressor mutation external to ben $A$ by being outcrossed to FGSC 99, a ts ${ }^{+}$wild-type strain. At least 50 segregants from each cross were tested for temperature sensitivity. The appearance of ts ${ }^{-}$segregants among the progeny of such a cross indicated that the reversion was caused by an indirect suppressor mutation and the absence of ts ${ }^{-}$segregants was taken to indicate that the reversion was probably caused by a backmutation in benA. Four revertants, benA17 R10, benA17 R11, benA17 R13 and benA21 R7, gave no ts ${ }^{-}$segregants and, therefore, probably represent back-mutations in ben $A$. The remaining 14 revertants all produced ts - progeny and must carry indirect suppressor mutations external to the ben $A$ gene (Table 3). 


\section{Table 3. Revertants of temperature-sensitive benA mutants}

Revertants were examined (i) for resistance (R) or sensitivity (S) to benomyl; (ii) for resistance or sensitivity to FPA; (iii) for temperature sensitivity at $44^{\circ} \mathrm{C}\left(\mathrm{ts}^{-}\right)$, cold sensitivity at $20^{\circ} \mathrm{C}(\mathrm{CS})$ or normal wild-type growth responses $\left(\mathrm{ts}^{+}\right)$[at least 50 segregants from each cross were tested]; (iv) for the presence of a suppressor $(\mathrm{Su})$ or back-mutation $(\mathrm{bm})$.

\begin{tabular}{|c|c|c|c|c|}
\hline Revertant strain & Benomyl & FPA & ts & $\mathrm{Su} / \mathrm{bm}$ \\
\hline BEN9 (benA11) & $\mathbf{R}$ & $\mathbf{R}$ & - & \\
\hline R1 & $\mathbf{R}$ & $\mathbf{R}$ & + & Su \\
\hline $\mathbf{R 7}$ & $\mathbf{R}$ & $\mathbf{R}$ & + & $\mathrm{Su}$ \\
\hline $\mathbf{R} 8$ & $\mathbf{R}$ & $\mathbf{R}$ & + & $\mathrm{Su}$ \\
\hline BEN15 (benA17) & $\mathbf{R}$ & $\mathbf{R}$ & - & \\
\hline R3 & $\mathbf{R}$ & $\mathbf{R}$ & + & $\mathrm{Su}$ \\
\hline R6 & $\mathbf{S}$ & $\mathbf{S}$ & CS & $\mathrm{Su}$ \\
\hline R7 & $\mathbf{R}$ & $\mathbf{S}$ & + & $\mathrm{Su}$ \\
\hline R8 & $\mathbf{R}$ & $\mathbf{R}$ & + & $\mathrm{Su}$ \\
\hline R9 & $\mathbf{R}$ & $\mathbf{S}$ & + & $\mathrm{Su}$ \\
\hline R10 & $\mathrm{S}$ & $\mathbf{S}$ & + & bm \\
\hline R11 & $\mathbf{R}$ & $\mathbf{R}$ & + & $\mathrm{bm}$ \\
\hline R12 & $\mathbf{R}$ & $\mathbf{S}$ & + & $\mathrm{Su}$ \\
\hline R13 & $S$ & $\mathbf{S}$ & + & bm \\
\hline BEN19 (benA21) & $\mathbf{R}$ & $\mathbf{R}$ & - & \\
\hline R7 & $\mathbf{R}$ & $\mathbf{R}$ & + & $\mathrm{bm}$ \\
\hline R8 & $\mathbf{R}$ & $\mathbf{R}$ & + & $\mathrm{Su}$ \\
\hline R9 & $\mathbf{R}$ & $\mathbf{R}$ & + & $\mathrm{Su}$ \\
\hline R10 & $\mathbf{R}$ & $\mathbf{R}$ & + & $\mathrm{Su}$ \\
\hline R11 & $\mathbf{S}$ & $\mathbf{S}$ & CS & $\mathrm{Su}$ \\
\hline R12 & $\mathbf{R}$ & $\mathbf{R}$ & + & $\mathrm{Su}$ \\
\hline
\end{tabular}

The ts ${ }^{+}$benA revertants were analysed for resistance to benomyl and to FPA and for temperature sensitivity (Table 3 ). In most cases reversion to $\mathrm{ts}^{+}$did not cause reversion to benomyl or FPA sensitivity. However, two extragenic suppressor strains, benA17 R7 and ben A17R9, were sensitive to FPA although still resistant to benomyl; and two other suppressor strains, benA17R6 and benA21R11, were sensitive to both benomyl and FPA. Two of the strains with probable back-mutations in benA, ben $A 17 \mathrm{R} 10$ and benA17 R13, were also revertant with respect to both drug resistance markers, whereas two other back-mutation strains, ben A17 R11 and ben A21 R7, remained resistant to both drugs. The fact that two of the back-mutations and two of the suppressor mutations caused the reversion not only of temperature sensitivity but also of benomyl and FPA resistance is convincing evidence that benomyl resistance, FPA resistance and temperature sensitivity are properties of the benA17 and benA21 mutations. By extrapolation it is likely that these are also properties of the benAll mutation.

The biochemical basis for FPA resistance has been described for three previously isolated FPA-resistant mutants of $A$. nidulans. Strains carrying the mutations $f p a A$ (tyr $A)$ and $f p a E(\operatorname{tryp} A)$ are resistant to FPA because they have defects in the pathways for aromatic amino acid biosynthesis which by a feedback mechanism elevate the intracellular concentration of phenylalanine, thereby reversing FPA toxicity. A strain carrying $f p a D$ is resistant to FPA because it has a permease defect which prevents the uptake of FPA (and phenylalanine) from the medium. Because mutations in tubulin might by some unknown mechanism affect intracellular amino acid levels or amino acid permease activity, we have compared phenylalanine and tyrosine levels in B3 and the ben A11, 17 and 21 strains and have also tested the ability of these strains to utilize extracellular phenylalanine.

To determine the relative phenylalanine and tyrosine levels of the wild-type and the three mutant strains, mycelia from overnight cultures were extracted with cold sulphosalicylic acid and subjected to amino acid analysis. The relative amounts of phenylalanine and tyrosine in the extracts were determined by comparing the areas of the absorption peaks for these 
Table 4. Ratios of phenylalanine and tyrosine to leucine in acid-soluble extracts of wild-type ben $\mathrm{A}^{+}$(B3) and temperature-sensitive ben A mutants (benA11, 17 and 21 ) of A. nidulans

The results show the ratios of the areas of the phenylalanine, leucine and tyrosine peaks from the amino acid analyser.

$\begin{array}{lcccc} & \text { B3 } & \text { benA11 } & \text { benA17 } & \text { benA21 } \\ \text { Phe/Leu } & 0 \cdot 23 & 0.22 & 0.28 & 0.22 \\ \text { Tyr/Leu } & 0.38 & 0.32 & 0.33 & 0.38\end{array}$

amino acids to the area of the absorption peak for leucine in the extracts (Table 4). In all three mutants the ratios of phenylalanine and tyrosine to leucine were essentially the same as in the wild-type parental strain B3. Thus, unless the concentration of leucine in these strains was abnormally elevated, which seems unlikely since the area of the leucine peak was normal with respect to all the other amino acids in the extracts, we can conclude that the FPA resistance associated with benA11, 17 and 21 is not caused by a high intracellular concentration of phenylalanine. To rule out the possibility that the temperaturesensitive ben $A$ mutations significantly decrease the uptake of FPA (or phenylalanine) from the medium, ben $A 21$ was crossed with a phenylalanine requiring (phen $A$ ) strain, FGSC 73. Approximately $50 \%$ of the benomyl-resistant segregants of the benA21 $\times$ FGSC 73 cross were phen $A$. Moreover, the benA phen $A$ segregants were indistinguishable from the ben $A$ phen $A^{+}$segregants with respect to colony size at phenylalanine concentrations between 0.05 and $100 \mu \mathrm{g} \mathrm{ml}^{-1}$, indicating that the double mutants were not limited in their ability to take up phenylalanine (and presumably FPA) from the medium. In contrast, the corresponding cross between $f p a D$ and phen $A$ has been shown to yield no fpaD phen $A$ segregants (Sinha, 1969), presumably because the double mutant can neither synthesize nor take up extracellular phenylalanine. Because of the difference between the results of these crosses, we consider it unlikely that the temperature-sensitive ben $A$ mutations cause FPA resistance by affecting amino acid uptake.

\section{DISCUSSION}

FPA is known to induce non-disjunction in fungi and to block mitosis in mammalian cells (Hastie, 1970; Sisken, 1973). Since FPA is structurally much simpler than most other drugs known to block mitosis, its mechanism of action is of some interest. In this paper, we have demonstrated that moderate resistance to FPA is caused by certain temperaturesensitive mutations in the gene for $\beta$-tubulin (ben $A)$ in $A$. nidulans. We have shown that FPA resistance cosegregates with ben $A$, and that back-mutations in ben $A$ and suppressors of benA cause reversion of FPA resistance.

Resistance to FPA has been studied extensively in A. nidulans (Sinha, 1967, 1969; Srivastava \& Sinha, 1975). Ten gene loci which cause FPA resistance have been identified previously in this organism and it has been estimated that mutation at as many as 28 different loci may cause FPA resistance (Srivastava \& Sinha, 1975). The mechanisms of resistance to FPA of the genes $f p a A, D$ and $E$ are known. FpaA and $E$ are blocked in tyrosine and tryptophan synthesis, respectively (Sinha, 1967). Normal metabolic control mechanisms cause organisms carrying these mutations to over-produce phenylalanine which then competes with FPA to prevent toxicity. The $f p a D$ mutation is known to interfere with amino acid uptake in $A$. nidulans (Sinha, 1969). We have determined that the intracellular pool of phenylalanine and the ability to utilize external phenylalanine are essentially normal in the temperature-sensitive ben $A$ mutants. Resistance to FPA is probably therefore an intrinsic property of these ben $A$ mutations in $\beta$-tubulin and not a consequence of an effect on metabolism or amino acid permease. This result suggests that FPA causes non-disjunction and blocks mitosis by directly affecting either the polymerization of tubulin or the function of microtubules in the mitotic spindle. It also implies that the site at which FPA acts may 
be related to the benomyl binding site on $\beta$-tubulin. Whether FPA acts as a ligand by binding to tubulin at this site or by being incorporated into tubulin in place of phenylalanine should be amenable to biochemical investigation.

This work was supported by grant no. GM 23060 from the National Institutes of Health.

\section{REFERENCES}

Clutterbuck, A. J. (1974). Aspergillus nidulans. In Handbook of Genetics, vol. 1, pp. 447-510. Edited by R. C. King. New York: Plenum Press.

DAVIDSE, L. C. (1973). Antimitotic activity of methyl benzimidazol-2-yl carbamate (MBC) in Aspergillus nidulans. Pesticide Biochemistry and Physio$\operatorname{logy} 3,317-325$.

DAVIDSE, L. C. \& FlaCh, W. (1977). Differential binding of methyl benzimidazol-2-yl carbamate to fungal tubulin as a mechanism of resistance to this antimitotic agent in mutant strains of Aspergillus nidulans. Journal of Cell Biology 72, 174-193.

HASTIE, A. C. (1970). Benlate-induced instability of Aspergillus diploids. Nature, London 226, 771-772.

LHOAS, P. (1961). Mitotic haploidization by treatment of Aspergillus niger diploids with $p$-fluorophenylalanine. Nature, London 190, 744-745.

Morris, N. R., LAI, M. H. \& OAKLeY, C. E. (1979). Identification of a gene for $\alpha$-tubulin in Aspergillus nidulans. Cell 16, 437-442.

Pontecorvo, C., Roper, J. A., Hemmons, L. M., MacDonald, K. D. \& Bufton, A. W. J. (1953). The genetics of Aspergillus nidulans. Advances in Genetics 5, 141-238.
Sheir-Neiss, G., LaI, M. H. \& Morris, N. R. (1978). Identification of a gene for $\beta$-tubulin in Aspergillus nidulans. Cell 15, 639-647.

SINHA, U. (1967). Aromatic amino acid biosynthesis and para-fluorophenylalanine resistance in Aspergillus nidulans. Genetical Research 10, 261-272.

SiNHA, U. (1969). Genetic control of the uptake of amino acids in Aspergillus nidulans. Genetics 62, 495-505.

SISKEN, J. E. (1973). The effects of $p$-D,L-fluorophenylalanine on chromosome movement and cytokinesis of human amnion cells in culture. Chromosoma 44, 91-98.

Srivastava, S. \& Sinha, U. (1975). Six new loci controlling resistance to $p$-fluorophenylalanine in Aspergillus nidulans. Genetical Research 25, 29-38.

VAN TUYL, J. M. (1977). Genetics of fungal resistance to systemic fungicides. Ph.D. thesis, Department of Phytopathology, Department of Genetics, Agricultural University, Wageningen, The Netherlands. 